\section{Law should recognize value of interspecies embryos}

SIR - Considerable time has been spent, during the current debate in the UK Parliament on the Human Fertilisation and Embryology Bill, on the definition and generation of interspecies embryos. A free vote is most likely on this part of the proposed legislation, which is perceived by some to be highly controversial.

We are among those proposing to undertake interspecies somatic-cell nuclear transfer - in which a human cell will be transferred into an animal egg. Our studies will generate embryos in order to advance understanding of genetic reprogramming and nuclear-mitochondrial interaction. They will also generate embryonic stem cells to model certain human diseases. As embryonic stem cells can differentiate into any type of body cell, those harbouring a specific genetic alteration can be differentiated into cells associated with the related disease and studied in vitro.

We acknowledge the exciting progress arising from induced pluripotent stem cells, whereby a fully differentiated cell is reprogrammed to behave like an undifferentiated stem cell. However, the success of this process is highly dependent on understanding embryo-derived stem cells. It remains to be determined whether induced pluripotent cells could lead to cancer or other diseases after transplantation, and whether they are equivalent to human embryonic stem cells, as they show different patterns of gene expression ${ }^{1}$. Studying reprogramming through somatic-cell nuclear transfer may improve induced pluripotency and help to produce therapeutically useful cells.

Animal oocytes are a far more reliable source than human oocytes, which are available in very limited supply. Embryonic stem cells have been derived following intraspecies nuclear transfer in mice ${ }^{2}$ and monkeys ${ }^{3}$, and several cell lines have resulted following transfer of human nuclei into rabbit oocytes ${ }^{4}$.

We strongly recommend that scientists be allowed to generate interspecies embryos and to culture these for up to 14 days, placing them under the same restrictions as any human embryos generated under current legislation. We also hope that the new legislation will be revised to allow researchers access to banks of well-characterized tissues and cells that were donated for research but not explicitly for the production of embryonic stem cells by somatic-cell nuclear transfer or other techniques. Such experiments should be conducted in parallel with those generating induced pluripotent cells, to compare the resultant cell lines and to learn more about reprogramming, nuclear-mitochondrial interaction and certain genetic diseases. It would be a retrograde step to prevent any avenue of research, especially one with such high potential gains.

Justin C. St John ${ }^{\star}$, Lyle Armstrong Stephen L. Mingert, Keith H. S. Campbells

*Mitochondrial and Reproductive Genetics Group, Clinical Sciences Research Institute, Warwick Medical School, Coventry CV2 2DX, UK $\uparrow$ Centre For Stem Cell Biology and Developmental Genetics, International Centre For Life, University of Newcastle Upon Tyne, Newcastle Upon Tyne NE1 3BZ, UK $\uparrow$ Stem Cell Biology Laboratory, Wolfson Centre for Age-Related Diseases, King's College London, London SE1 1UL, UK

§Animal Development and Biotechnology Group, School of Biosciences, University of Nottingham, Sutton Bonington, Loughborough, Leicestershire LE12 5RD, UK

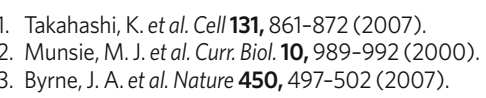

\section{Italian neuroscientists are ready to start the debate}

SIR - Neurotechnologies such as cognitive enhancement (see Nature 450, 1157-1159; 2007 and Nature 451, 520-521; 2008) and brain reading are likely to have huge social impact. The ethical problems connected with their expected development demand public discussion in our communities. Neuroscientists should take an active part in this debate, as you commented in your Editorial 'Neuroethics needed' (Nature 441, 907; 2006).

We therefore drafted a questionnaire for the 703 members of the Italian Society of Neuroscience (see tinyurl.com/2pwehh). Among other questions, we asked about their interest in neuroethics issues; specific topics discussed in their labs; how they stay up to date; the amount of related reading they do; what specialists they believe are engaged in neuroethics and who they think should be; and how they rate handling of neuroethics issues by the Italian media.

Ten per cent of those surveyed responded. This sample did not vary significantly from the surveyed population with respect to age, discipline or affiliation $(P<0.05)$. The answers showed that $91 \%$ (95\% confidence interval: 84-98) of respondents are interested in neuroethics; 78\% (95\% confidence interval: 68-88) believe that neuroethics problems should be tackled in collaboration with bioethicists and neuroscientists; and 96\% (95\% confidence interval: 91-100) would be willing to take part in further initiatives. Neuroscientists under the age of 35 seemed to be the least informed.
Although the small number of responses may indicate a lack of awareness among neuroscientists in Italy about neuroethical issues, the responders show that a significant and representative proportion are interested in public debate.

We offer these results to the community in the hope that our initiative can be replicated on a larger scale. We hope that Italy's media and politicians will exploit the availability of neuroscientists to discuss scientific problems of such outstanding social interest.

Fiorenzo Conti ${ }^{\star}$, Gilberto Corbellini†

*Department of Neuroscience, Università

Politecnica delle Marche, Via Tronto 10/A, 60026 Ancona, Italy

$\uparrow$ Department of Experimental Medicine, Section of History of Medicine, Viale dell'Università 34/a, 00185 Roma, Italy

\section{Darwin's legacy makes its mark in Croatia}

SIR - Charles Darwin's On the Origin of Species and The Descent of Man have at last been translated into Croatian, thanks to the work of the renowned science and theology translator Josip Balabanić. Other European countries - including Denmark, the Netherlands, France, Germany, Italy, Poland, Russia and Sweden - had access to Darwin's works in their mother tongue during his lifetime. But it was not until this year that Croatian students of biology could read them in their own language.

Religious education was introduced in elementary schools during the early years of Croatian independence, and ethics and the major world religions are now studied in high school. At the same time, the importance of evolution for modern biology and medicine is publicly acknowledged by science academies and societies - in the spirit of your Editorial 'Spread the word' (Nature 451, 108; 2008).

Croatia aspires to join the group of countries in which education and science occupy prime positions in national strategies, and recognizing the influence of Darwin's writings is an important step in that direction. Celebrations of the 200th anniversary of Darwin's birth on 12 February next year, possibly at the Croatian Academy of Sciences and Arts, will have particular significance for Croatians.

Jasmina Muzinic

Department of Ornithology, Croatian Academy of Sciences and Arts, Gunduliceva 24, HR-10000 Zagreb, Croatia

\footnotetext{
Contributions to Correspondence may be submitted to correspondence@nature.com. They should be no longer than $\mathbf{3 0 0}$ words, and preferably shorter. We welcome comments on publishing issues at Nautilus (http://blogs.nature.com/nautilus).
} 